\title{
Speech Therapy Mobile Application for Speech and Language Impairment Children
}

\author{
Carolyne Alphonsus Tommy \\ Institute of Social Informatics and Technological Innovations, \\ Universiti Malaysia Sarawak, \\ Kota Samarahan, Sarawak, Malaysia
}

\author{
Jacey-Lynn Minoi \\ Faculty of Computer Science and Information Technology, \\ Universiti Malaysia Sarawak, \\ Kota Samarahan, Sarawak, Malaysia
}

\begin{abstract}
Speech and language impairment (SLI) is a communication disorder when a person has difficulties to produce speech sounds correctly and understand others. Children with SLI need speech therapy to help them improve their communication skills in order to communicate effectively with others. Due to the lack of speech therapist in the state, children are mainly waste valuable learning time while waiting for therapy and these speech therapy sessions are usually done only on a one-to-one basis within a block period. Recent studies showed that mobile technologies may work as an educational tool which can help children to learn. Hence, a local prototype of mobile speech therapy application was developed to be appealing for pre-school children of age 3 to 6 with SLI and will be used as a supplementary activity which can be used during a speech therapy session or at home. In this paper, we present the results of a six months intervention experiment with SLI children. We have adapted the heuristic evaluation for evaluating the usability of the local prototype mobile speech therapy application. The results of our study shows those children and their parents gave positive responses towards the mobile application, also helps in improving the children skills in producing correct sounds.
\end{abstract}

Keywords - mobile application; speech therapy; speech and language impairment (SLI)

\section{INTRODUCTION}

Speech and Language Impairment (SLI) is a communication disorder that occurs in a person's ability to talk, read, write and understand others. Language impairment is the difficulty in understanding the language and the processes, such as vocabulary or grammar ([1], [2]). Speech is often referred to articulation, and the disorder of speech or articulation problem arises when a child has a difficulty in producing the correct sounds, syllables and words. Children experiencing speech production impairment would impact their articulation and speech intelligibility [3], which risk future reading and communication skills. If unresolved, it could cause difficulties of both learning and socialisation and lasting into adolescence and beyond [4].
Speech therapy will be needed as a treatment programme to help those children with SLI by speechlanguage pathology (SLP). However, in Malaysia, there is still lack of qualified SLP. This can be found in the updated list of qualified speech and language therapist based on the members' directory kept by Malaysian Association of Speech-Language \& Hearing (MASH) [5]. Given that children nowadays are easily accessible and exposed to mobile technology at a younger age. Hence, integrating technology into speech and language therapy can be used as an alternative approach of speech therapy since technology has become more accessible to everyone. This paper will discuss the usability of the first version of local prototype mobile speech therapy application developed based on the results gathered from heuristic evaluation that was conducted with pre-school children with speech delay.

\section{WHAT IS SPEECH THERAPY?}

Speech therapy is a clinical program conducted by speech therapist or SLP as a treatment programme to help SLI children. As defined by American Speech-Language Hearing Association (ASHA) in Speech-Language Pathology Medical Review Guidelines [6], SLP helps by providing treatment to those with disorders of speech sound production, resonance, voice, fluency, language, cognition and feeding and swallowing. SLPs aim to implement an efficient treatment programmes to help resolves children's spoken difficulties as well as to prevent later literacy problems [7]. SLP has to go through certain assessments to collect information about the behaviour and progress of the children with SLI. The assessments include understanding of spoken language and body language of the children, children's expression through speaking and body language, production and usage of sounds, their ability to use language in a social context, play skills and eating, drinking and swallowing. The treatment programme approach comprises of therapy manual, involving object-based and paper-based method. This includes a set of pictures or flashcards to represent any single consonants, vowels and words too. Those pictures or flashcards are presented to the children and are asked to produce sounds or words based on the pictures. 